\title{
Comparative Analysis of Energy Efficient Routing Protocols in MANET
}

\author{
Mohammad Rafiq \\ Asst.Professor (CSE) \\ Islamic University, Awantipora \\ J\&K, India
}

\author{
Manoj Kumar \\ Research Scholar (Ph.D-CS) \\ Shobhit University, Meerut \\ U.P, India
}

\author{
Shabeer Ahmad \\ Asst.Professor (E\&C) \\ Islamic University, Awantipora \\ J\&K, India
}

\begin{abstract}
A Mobile Ad Hoc Network (MANET) is a collection of wireless mobile nodes forming a temporary/short-lived network without any fixed infrastructure where all nodes are free to move about arbitrarily and where all the nodes configure themselves. This paper focus on the energy consumption issues of the routing protocols. This paper examines the energy consumption behavior of three routing protocols; Ad hoc On Demand Distance Vector (AODV), Dynamic Source Routing (DSR) and the Destination Sequenced Distance Vector Routing (DSDV) with respect to energy consumption. Evaluating how the different approaches and algorithms affect the energy usage in the mobile devices. This paper analyses these routing protocols by extensive simulations in ns- 2 simulator and show that how the number of nodes affect their performance. In this study performance is measured in terms of Average Remaining Energy, Average Consumed Energy, Network Life Time, System Life Time and Energy Consumption per successful data delivery .
\end{abstract}

\section{Keywords}

AODV, DSDV, DSR, MANET, Routing, Protocols, Energy Consumption.

\section{INTRODUCTION}

Wireless networks emerged in the 1970's, since then they have become increasingly popular. The reason of their popularity is that they provide access to information regardless of the geographical location of the user. Ad-hoc networks are a new paradigm of wireless communication for mobile hosts. No fixed infrastructure such as base stations as mobile switching. Nodes within each other radio range communicate directly via wireless links while those which are far apart, rely on other nodes to relay messages. In ad-hoc networks, node mobility causes frequent changes in topology. Also there are some parameters i.e. (absence of centralized control, each node has wireless interface, nodes can move around freely which results in frequent changes in network topology, nodes have limited amount of resources and lack of symmetrical links i.e. transmission does not usually perform equally well in both directions) which make them dynamic in nature. In MANET, each node acts both as a router and as a host and even the topology of network may also change rapidly. These types of networks assume existence of no fixed infrastructure [1]. A MANET is a type of ad hoc network that can change locations and configure itself on the fly. Because MANET is mobile, they use wireless connections to connect to various networks. This can be a standard Wi-Fi connection, or another medium, such as a cellular or satellite transmission. MANET contains a special subset of wireless networks since they do not require the existence of a centralized message-passing device. In MANETs all network activities including topology discovery and delivering messages must be executed by nodes themselves. Hence it is said that an adhoc network is decentralized. MANET comprises of mobile router. MANETs are able to operate in a stand-alone fashion or could possibly to a larger network such as internet The Communication in MANET takes place by using multi-hop paths. The mobile hosts can move randomly and can be turned on or off without notifying other hosts. If two wireless hosts are out of their transmission ranges in the ad hoc networks, other mobile hosts placed between them can forward their messages, which effectively build connected networks among the mobile hosts in the deployed area.

\section{FEATURES OF MANET}

A Mobile Ad hoc Network has the following features [2]:

Autonomous terminal: In MANET, each mobile terminal is an autonomous node, which may function as both a host and a router. In other words, besides the basic processing ability as a host, the mobile nodes can also perform switching functions as a router. So usually endpoints and switches are indistinguishable in MANET.

Distributed operation: Since there is no background network for the central control of the network operations, the control and management of the network is distributed among the terminals. The nodes involved in a MANET should collaborate amongst themselves and each node acts as a relay as needed, to implement functions e.g. security and routing.

Multihop routing: Basic types of ad hoc routing algorithms can be single-hop and multihop, based on different link layer attributes and routing protocols. Single-hop MANET is simpler than multihop in terms of structure and implementation, with the cost of lesser functionality and applicability. When delivering data packets from a source to its destination out of the direct wireless transmission range, the packets should be forwarded via one or more intermediate nodes.

Dynamic network topology: Since the nodes are mobile, the network topology may change rapidly and unpredictably and the connectivity among the terminals may vary with time. MANET should adapt to the traffic and propagation conditions as well as the mobility patterns of the mobile network nodes. The mobile nodes in the network dynamically establish routing among themselves as they move about, forming their own network on the fly. Moreover, a user in the MANET may not only operate within the ad hoc network, but may require access to a public fixed network (e.g. Internet).

Fluctuating link capacity: The nature of high bit-error rates of wireless connection might be more profound in a MANET. One end-to-end path can be shared by several sessions. The channel over which the terminals communicate is subject to noise, fading, and interference, and has less bandwidth than a 
wired network. In some scenarios, the path between any pair of users can traverse multiple wireless links and the link themselves can be heterogeneous.

Light-weight terminals: In most cases, the MANET nodes are mobile devices with less CPU processing capability, small memory size, and low power storage. Such devices need optimized algorithms and mechanisms that implement the computing and communicating functions.

\section{MANET Challenges}

The following list of challenges shows the inefficiencies and limitations that have to be overcome in a MANET environment [3].

Limited wireless transmission range: In wireless networks the radio band will be limited and hence data rates it can offer are much lesser than what a wired network can offer. This requires the routing protocols in wireless networks to use the bandwidth always in an optimal manner by keeping the overhead as low as possible. The limited transmission range also imposes a constraint on routing protocols in maintaining the topological information. Especially in MANET's due to frequent changes in topology, maintaining the topological information at all nodes involves more control overhead which, in turn, results in more bandwidth wastage.

Packet loss due to transmission errors: Ad hoc wireless networks experiences a much higher packet loss due to factors such as high bit error rate (BER) in the wireless channel, increased collisions due to the presence of hidden terminals, presence of interference, location dependent contention, unidirectional links, frequent path breaks due to mobility of nodes, and the inherent fading properties of the wireless channel.

Mobility-induced route changes: The network topology in an ad hoc wireless network is highly dynamic due to the movement of nodes; hence an on-going session suffers frequent path breaks. This situation often leads to frequent route changes. Therefore mobility management itself is very vast research topic in ad hoc networks.

Mobility-induced packet losses: Communication links in an ad hoc network are unstable such that running conventional protocols for MANETS over a high loss rate will suffer from severe performance degradation. However, with high error rate, it is very much difficult to deliver a packet to its destination.

Battery constraints: This is one of the limited resources that form a major constraint for the nodes in an ad hoc network. Devices used in these networks have restrictions on the power source in order to maintain portability, size and weight of the device. By increasing the power and processing ability makes the nodes bulky and less portable. So, only MANET's node has to optimally use this resource.

Potentially frequent network partitions: The randomly moving nodes in an ad hoc network can lead to network partitions. In major cases, the intermediate nodes are the one which are highly affected by this partitioning.

Ease of snooping on wireless transmissions (security issues): The radio channel used for ad hoc networks is broadcast in nature and is shared by all the nodes in the network. Data transmitted by a node is received by all the nodes within its direct transmission range. So an attacker can easily snoop the data being transmitted in the network. Here the requirement of confidentiality can be violated if an adversary is also able to interpret the data gathered through snooping.

\section{ROUTING PROTOCOLS}

In this section we briefly review the studied routing protocols.

\subsection{Ad Hoc on-Demand Distance Vector Routing (AODV)}

AODV provides on-demand route discovery in MANET. Whenever the nodes need to send data to the destination, if the source node doesn't have routing information in its table, route discovery process begins to find the routes from source

to destination. Route discovery begins with broadcasting a route request (RREQ) packet by the source node to its neighbors. RREQ packet comprises broadcast ID, two sequence numbers, the address of source and destination and hop count. The intermediary nodes which receive the RREQ packet could do two steps: If it isn't the destination node then it'll rebroadcast the RREQ packet to its neighbors.

Otherwise it'll be the destination node and then it will send a unicast replay message, route replay (RREP), directly to the source from which it was received the RREQ packet. A copied RREQ will be ignored. Each node has a sequence number. When a node wants to initiate route discovery process, it includes its sequence number and the most fresh sequence number it has for destination. The intermediate node that receive the RREQ packet, replay to the RREQ packet only when the sequence number of its path is larger than or identical to the sequence number comprised in the RREQ packet. A reverse path from the intermediate node to the source forms with storing the node's address from which initial copy of RREQ. There is an associated lifetime value for every entry in the routing table. Suppose that some routes are not applied within their lifetime period, so these routes are expired and should be dropped from the table. But if routes are used, the lifetime period is updated so those routes are not expired. When a source node wants to send data to some destination, first it searches the routing table; if it can find it, it will use it. Otherwise, it must start a route discovery to find a route [4]. It is also Route Error (RERR) message that used to notify the other nodes about some failures in other nodes or links.

\subsection{DSR - Dynamic Source Routing (DSR)}

DSR is a reactive routing protocol that discovers and maintains routes between nodes. In the route discovery, DSR floods Route Request Packet to the network [4]. Each node that receives this packet, first add its address to it and then forwards the packet to the next node. When the targeted node or a node that has route to the destination receives the Route Request, it returns a Route Reply to the sender and a route is established. Each time a packet follows an established route, each node has to ensure that the link is reliable between itself and the next node. In the Route maintenance, DSR provides three successive steps: link layer acknowledgment, passive acknowledgment and network layer acknowledgment. When a route is broken and one node detects the failure, it sends a Route Error packet to the original sender

\subsection{Destination-Sequenced Distance-Vector Routing}

The Destination-Sequenced Distance-Vector Routing protocol (DSDV) described in is a table-driven algorithm based on the classical Bellman-Ford routing mechanism [1]. The 
improvements made to the Bellman-Ford algorithm include freedom from loops in routing tables. Every mobile node in the network maintains a routing table in which all of the possible destinations within the network and the number of hops to each destination are recorded. Each entry is marked with a sequence number assigned by the destination node. The sequence numbers enable the mobile nodes to distinguish stale routes from new ones, thereby avoiding the formation of routing loops. Routing table updates are periodically transmitted throughout the network in order to maintain table consistency. To help alleviate the potentially large amount of network traffic that such updates can generate, route updates can employ two possible types of packets. The first is known as a full dump. This type of packet carries all available routing information and can require multiple network protocol data units (NPDUs). During periods of occasional movement, these packets are transmitted infrequently. Smaller incremental packets are used to relay only that information which has changed since the last full dump. Each of these broadcasts should fit into a standard-size NPDU, thereby decreasing the amount of traffic generated. The mobile nodes maintain an additional table where they store the data sent in the incremental routing information packets. New route broadcasts contain the address of the destination, the number of hops to reach the destination, the sequence number of the information received regarding the destination, as well as a new sequence number unique to the broadcast. The route labeled with the most recent sequence number is always used. In the event that two updates have the same sequence number, the route with the smaller metric is used in order to optimize (shorten) the path. Mobiles also keep track of the settling time of routes, or the weighted average time that routes to a destination will fluctuate before the route with the best metric is received.

\section{SIMULATION PERFORMED USING} NS-2

We have used Network Simulator (NS)-2 in our evaluation. The NS-2 is a discrete event driven simulator developed at UC Berkeley. NS-2 is suitable for designing new protocols, comparing different protocols and traffic evaluations. It is an object oriented simulation written in $\mathrm{C}++$, with an OTcl interpreter as a frontend. Simulation of protocols is performed on Linux operating system using ns-2.34. We have different simulations run in all over. Every simulation runs from 0s to 200s. Random waypoint mobility in a rectangular field of $400 \mathrm{~m} * 400 \mathrm{~m}$ is used.

\subsection{Simulation Steps}

1) Scenarios are generated using setdest utility which uses random waypoint mobility model. Example to generate scenario is given as:

Setdest -v1 -n 30 - p 0.0 - M 4 -t 200 -x 400 -y 400 > scene5

Where $-\mathrm{v}$ : version 1 or $2,-n$ : number of nodes , -p : pause time , $-\mathrm{M}$ : maximum speed, $-\mathrm{x}$ and $-\mathrm{y}$ : area of

simulation, - $t$ : simulation time, scene5 : output file.

2) Traffic pattern is generated using cbrgen.tcl file given in indep utilities. In this simulation only one traffic pattern is generated using following method:

ns cbrgen.tcl - type cbr -nn 9 - seed 1 - mc 7 - rate 4 > rafiq7

Where - type: type of traffic cbr or tcp, - nn: number of nodes, - seed: seed value, -mc: maximum connection sources, -rate: rate of sending packets.
3) After generating traffic patterns and scenarios a tcl script is written for the generation of the trace files. These created traffic patterns and scenarios are fed in to tcl script and then executed. On execution of tcl script trace files are generated. In this simulation two protocols namely AODV,DSR and DSDV are used to generate trace files which are saved with the extension *.tr which are old trace file formats. There are two trace file formats available one is old trace file format and other is new trace file format. With generation of trace file a *.nam file is also generated which shows animation of the moving nodes and routing of packets. Routing of packets and movement of nodes can be easily depicted by *.nam files.

4) When trace files are generated then it is needed to analyse these files. To analyse files awk or perl scripts are written according to performance metrics which are to be used in performance evaluation. This simulation is performed to evaluate the performance based on metrics namely Average Remaining Energy, Average Consumed Energy, Network Life Time , System Life Time and ECSDD . So five awk files are used for this simulation.

5) After the analysis of trace files obtained results are stored in a *.xgr file from which $\mathrm{x}$ graphs are generated by using Xgraph utility of ns-2.

\subsection{Performance Metrics}

There are many performance metrics which are used for analysis of various protocols. In this project I am using four performance metrics, which are:

Average Remaining Energy/Average System Energy: It is taken as the average of the remaining energy levels of all the nodes in the network at the end of simulation.

Average Consumed Energy: It is taken as the average of the consumed energy of all the nodes in the network at the end of simulation.

Network Life Time: It is the time when a node finished its own battery for the first time. The performance is better when network life time is high [5].

System Life Time: It is the time when $20 \%$ of nodes finish their own battery. The performance is better when system life time is high [5].

ECSDD (Energy Consumption per Successful Data Delivery): It is the ratio of total energy consumption to the number of data packets successfully delivered to the destination . Lower the ECSDD values indicate that node uses less energy for data communication. This helps in extending the lifetime of node and thus overall network lifetime.

\subsection{Simulations and Results}

The simulations were performed using Network Simulator 2 (Ns-2), particularly popular in the ad hoc networking community. The mobility model used is Random Way point Model. The traffic sources are CBR (continuous bit rate), number of data connections is 7, data packet size is 512 byte and data sending rate is 4 packet/second. The source destination pairs are spread randomly over the network in a rectangular filed of $400 \mathrm{~m} \times 400 \mathrm{~m}$. During the simulation, each node starts its journey from a random spot to a random chosen destination. The simulation time is 200 seconds and maximum speed of nodes is $20 \mathrm{~m} / \mathrm{s}$. The interface queue is 50 packet drop-tail priority queue. Network scenario for different number of nodes are generated. Impact of number of nodes In this simulation number of nodes is varying and considered 10 , 
$15,20,25$ and 30 and other network parameters are considered as in the table 1 .

Table -1 Simulation Parameters

\begin{tabular}{|c|c|}
\hline Parameters & Values \\
\hline Routing Protocols & AODV,DSDV,DSR \\
\hline Simulation time & $200 \mathrm{sec}$ \\
\hline Traffic Type & CBR \\
\hline Maximum Connections & 7 \\
\hline Sources & 5 \\
\hline Packet Rate & 4 (pkts/sec) \\
\hline Packet Size & 512 bytes \\
\hline Pause Time & 0 \\
\hline Number of nodes & $10,15,20,25,30$ \\
\hline Network Area & $400 \times 400 \mathrm{~m}^{2}$ \\
\hline Transmission Range & $250 \mathrm{~m}$ \\
\hline Maximum Speed & $20 \mathrm{~m} / \mathrm{sec}$ \\
\hline Mobility Model & Random Waypoint \\
\hline Interface Queue & $\begin{array}{l}50 \text { Packet Drop-tail } \\
\text { Priority }\end{array}$ \\
\hline Mac type & IEEE 802_11 \\
\hline Initial energy & $\begin{array}{l}200 \mathrm{~J} \text { ( } 146 \mathrm{~J} \text { in case of } \\
\text { network and system life } \\
\text { Time) }\end{array}$ \\
\hline Idle power & $0.73 \mathrm{~W}$ \\
\hline Tx power & $1.4 \mathrm{~W}$ \\
\hline Rx power & $0.9 \mathrm{~W}$ \\
\hline Sleep power & $0.001 \mathrm{~W}$ \\
\hline Transition power & $0.1 \mathrm{~W}$ \\
\hline Transition time & $0.005 \mathrm{sec}$ \\
\hline Antenna Type & Omni-Directional \\
\hline
\end{tabular}

We simulated this network under each of routing protocols and outputs shown in Figs. 1-6. Figs. 1-6 show a comparison between the routing protocols as a function of number of nodes.

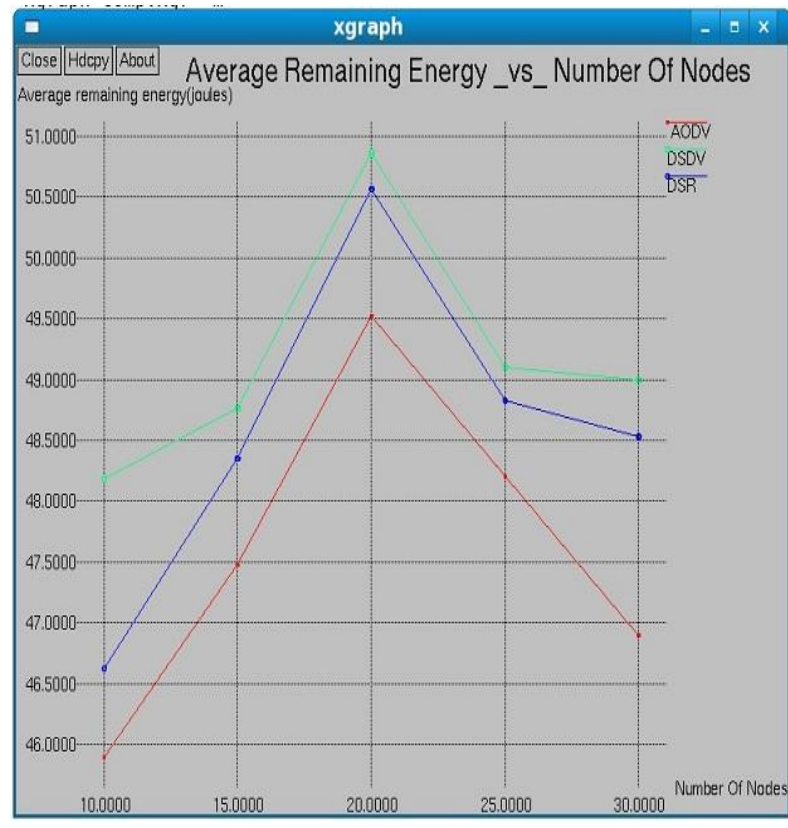

Figure 1.Average Remaining Energy versus Number of nodes .

Figures 1-2 shows that Average Remaining energy and \% Average System Energy of DSDV is higher than DSR and AODV when we are increasing number of nodes from 10 to 30 for this network.

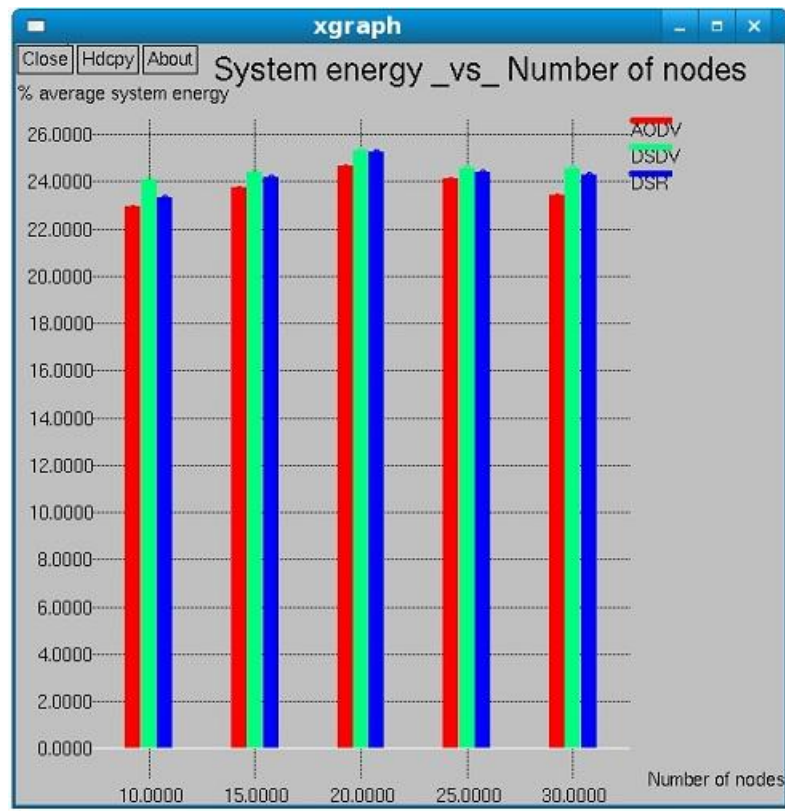

Figure 2. \% Average System Energy vs Number of nodes

Figure 3 shows that Average Consumed energy of AODV is higher than DSR and DSDV when we are increasing number of nodes from 10 to 30 for this network. 


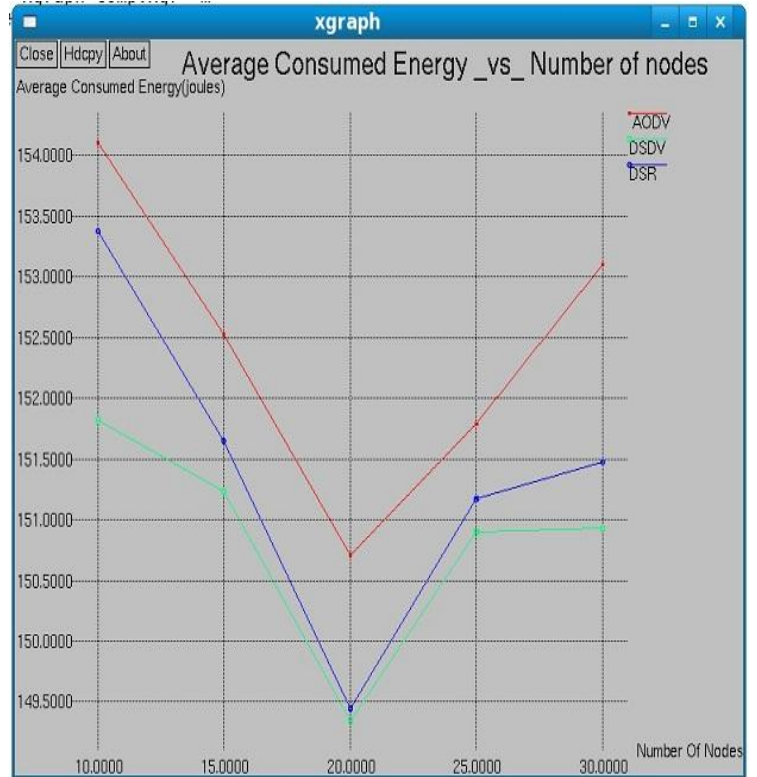

Figure 3. Average Consumed Energy vs Number of nodes .

Figures 4-5 shows that Average Network Life Time and System Life Time of DSDV is higher than DSR and AODV when we are increasing number of nodes from 10 to 30 for this network.

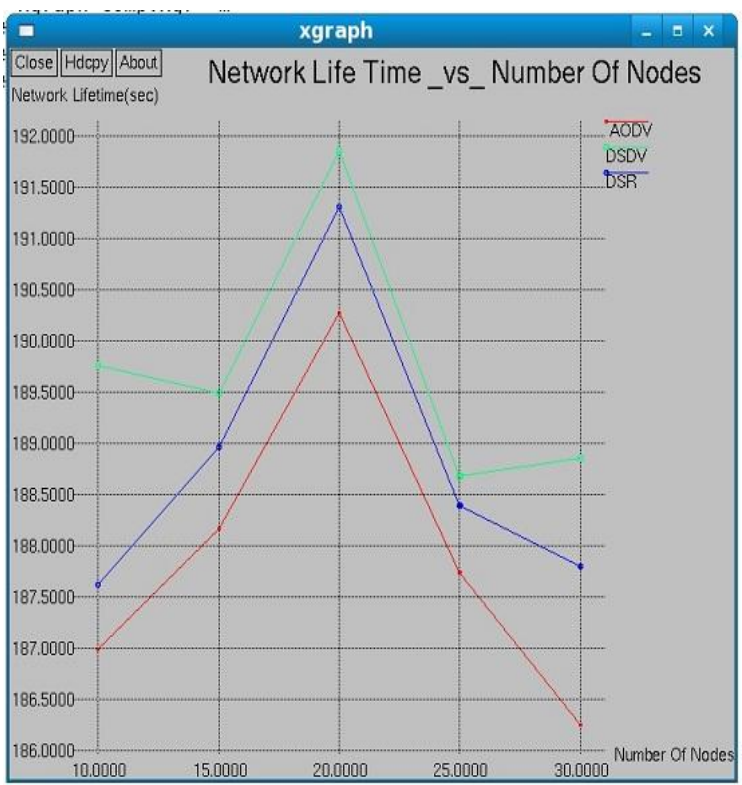

Figure 4. Network Life Time vs Number of nodes .

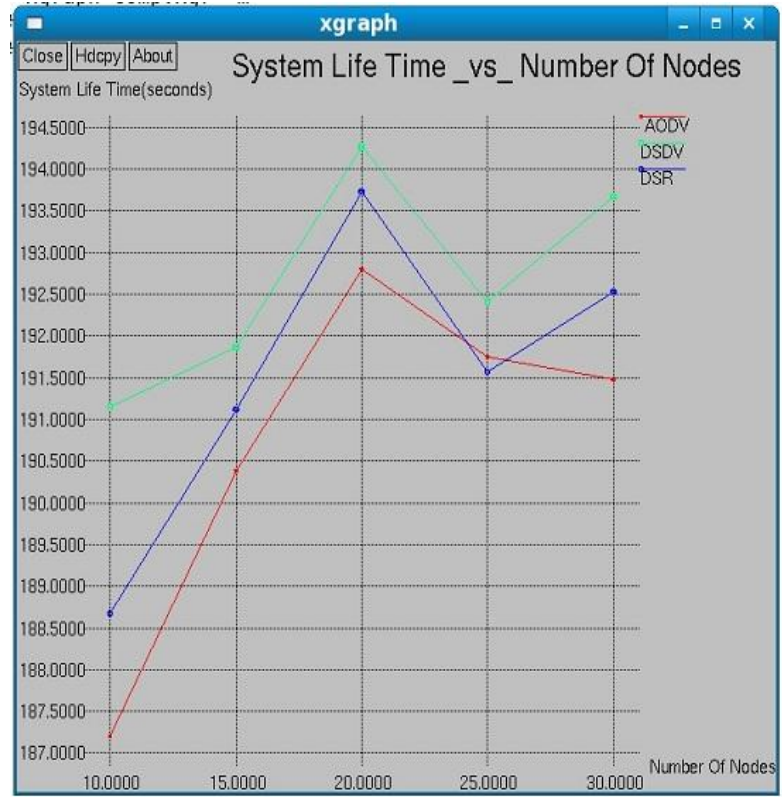

Figure 5. System Life Time vs Number of nodes .

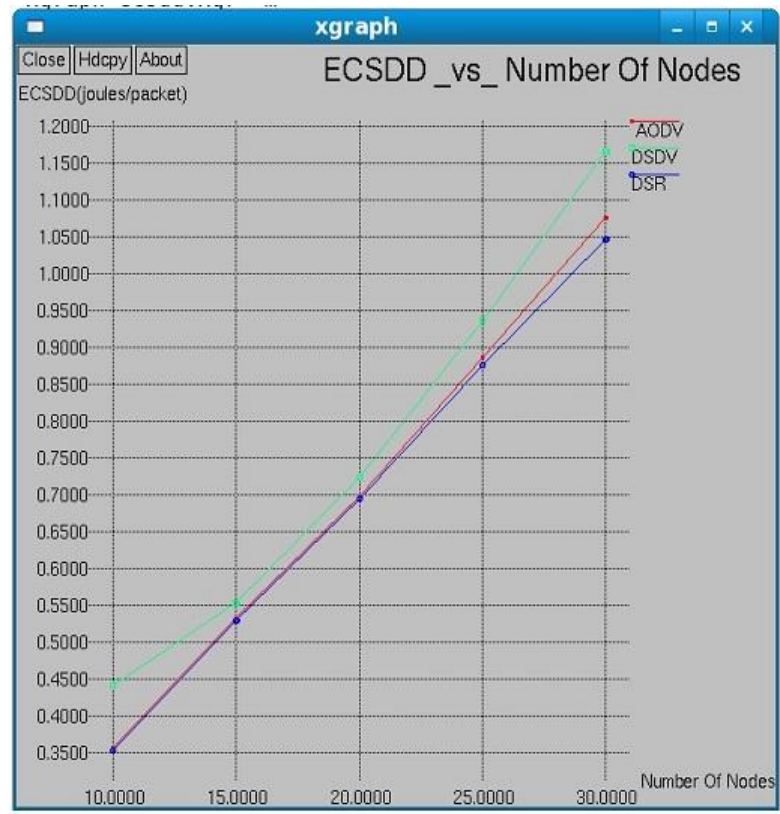

Figure 6. ECSDD vs Number of nodes.

Figure 6 shows effect of increasing node density on ECSDD and ECSDD increases for all the three considered routing protocols when we are increasing number of nodes and DSDV has maximum ECSDD for this network .DSDV consumes less energy but ECSDD is more for DSDV which means it allows less successful data delivery to destination. 


\section{CONCLUSION}

This paper is an attempt to evaluation performance of Three commonly used mobile ad hoc routing protocols namely AODV, DSDV and DSR. Performance evaluation performed in NS-2 simulator by doing many simulations. Comparison was based on Average Remaining Energy, Average Consumed Energy, Network Life Time, System Life and ECSDD. Simulation results are shown by many figures. By using simulation results we can understand that DSDV gives better performance in wide range of simulation conditions for this network but ECSDD should be less but it is high for DSDV.

\section{REFERENCES.}

[1] Beigh Bilal Maqbool and Prof. M.A.Peer "Classification of Current Routing Protocols for Ad Hoc Networks - A Review" International Journal of Computer Applications Volume 7- No.8, October 2010.

[2] Manijeh Keshtgary and Vahide Babaiyan, "Performance Evaluation of Reactive, Proactive and Hybrid Routing Protocols in MANET " International Journal on Computer Science and Engineering (IJCSE). Vol. 4 No. 02 February 2012.

[3] Jun-Zhao Sun, "Mobile Ad Hoc Networking: An Essentia 1Technology for Pervasive Computing", Infotech and Info-net, vol.3, 2001, pp316-321.

[4] G. S. Mamatha and Dr. S. C. Sharma, "Analyzing the MANET Variations, Challenges, Capacity and Protocol Issues", International Journal of Computer Science \& Engineering Survey (IJCSES) Vol.1, No.1, August 2010.
[5] Mina Vajed Khiavi, Shahram Jamali, Sajjad Jahanbakhsh Gudakahriz "Performance Comparison of AODV, DSDV, DSR and TORA Routing Protocols in MANETs" International Research Journal of Applied and Basic Sciences. Vol., 3 (7), 1429-1436, 2012.

[6] Bilal Maqbool, Dr. M.A.Peer, and S.M.K.Quadri "Towards the Benchmarking of Routing Protocols for Adhoc Wireless Networks" IJCST Vol. 2, Iss ue 1, March 2011.

[7] Dinesh Singh, Deepak Sethi, Pooja “ Comparative Analysis of Energy Efficient Routing Protocols in MANETS (Mobile Ad-hoc Networks)" International Journal of Computer Science and Technology Vol. 2, Issue 3, September 2011

[8] K. Arulandam and Dr.B.Parthasarathy "A new energy level efficiency issues in MANET" International Journal of Reviews in Computing 2009.

[9] Mobile communications by $\mathbf{J}$ Schiller pearson $2^{\text {nd }}$ editon.

[10] Ad Hoc Networking, Perkins, Addison Wesley, 2001.

[11] Marc Greis, Ns Tutorial, http://www.isi.edu /nsnam/ns/tutorial/, accessed on Sep 2012

[12] TCL Tutorial, http://www.tcl.tk /man/tcl/tutorial/ tcltutorial.html, accessed on Sep 2012.

[13] Trace formats, http://nsnam.isi.edu/nsnam/index.php/NS2_Trace_Formats , accessed on Jan 2013

[14] Awk basics ,http://www.thegeekstuff .com/2010/01/ awk-introduction -tutorial-7-awk-print-examples/ , accessed on Jan2013 